\title{
Regulation of infection efficiency in a globally abundant marine Bacteriodetes virus
}

\author{
Cristina Howard-Varona ${ }^{1,6}$, Simon Roux ${ }^{2,6}$, Hugo Dore ${ }^{3}$, Natalie E Solonenko ${ }^{2,6}$, \\ Karin Holmfeldt ${ }^{4}$, Lye M Markillie ${ }^{5}$, Galya Orr ${ }^{5}$ and Matthew B Sullivan ${ }^{1,2,6,7}$ \\ ${ }^{1}$ Department of Molecular and Cellular Biology, University of Arizona, Tucson, AZ, USA; ${ }^{2}$ Department of \\ Ecology and Evolutionary Biology, University of Arizona, Tucson, AZ, USA; ${ }^{3}$ Département de biologie, ENS \\ Lyon, Lyon, France; ${ }^{4}$ School of Natural Sciences, Linnaeus University, Kalmar, Sweden and ${ }^{5}$ Environmental \\ Molecular Sciences Laboratory, Pacific Northwest National Laboratory (PNNL), Richland, WA, USA
}

\begin{abstract}
Bacteria impact humans, industry and nature, but do so under viral constraints. Problematically, knowledge of viral infection efficiencies and outcomes derives from few model systems that overrepresent efficient lytic infections and under-represent virus-host natural diversity. Here we sought to understand infection efficiency regulation in an emerging environmental Bacteroidetes-virus model system with markedly different outcomes on two genetically and physiologically nearly identical host strains. For this, we quantified bacterial virus (phage) and host DNA, transcripts and phage particles throughout both infections. While phage transcriptomes were similar, transcriptional differences between hosts suggested host-derived regulation of infection efficiency. Specifically, the alternative host overexpressed DNA degradation genes and underexpressed translation genes, which seemingly targeted phage DNA particle production, as experiments revealed they were both significantly delayed (by $>30 \mathrm{~min}$ ) and reduced (by $>50 \%$ ) in the inefficient infection. This suggests phage failure to repress early alternative host expression and stress response allowed the host to respond against infection by delaying phage DNA replication and protein translation. Given that this phage type is ubiquitous and abundant in the global oceans and that variable viral infection efficiencies are central to dynamic ecosystems, these data provide a critically needed foundation for understanding and modeling viral infections in nature.
\end{abstract}

The ISME Journal (2017) 11, 284-295; doi:10.1038/ismej.2016.81; published online 17 May 2016

\section{Introduction}

Bacteria are well recognized to impact human health and disease (Heintz and Mair, 2014), industrial processes (Luo et al., 2015) and natural ecosystems (Falkowski et al., 2008). More recently, viruses are also gaining relevance as they modulate these processes through cell lysis, horizontal gene transfer and metabolic reprogramming during infection (Fuhrman, 1999; Wommack and Colwell, 2000; Hurwitz et al., 2013; Salmond and Fineran, 2015). Although most bacteria are thought to be infected by viruses (phages) (Weinbauer, 2004; Hyman and Abedon, 2012), and the extent of viral impacts depends on the efficiency (e.g., fraction of infected/

Correspondence: MB Sullivan Sullivan, Department of Microbiology and Civil, Environmental and Geodetic Engineering, The Ohio State University, Columbus, $\mathrm{OH} 43210$, USA.

E-mail: mbsulli@gmail.com

${ }^{6}$ Current address: Department of Microbiology, The Ohio State University, Columbus, OH, USA.

${ }^{7}$ Current address: Department of Civil, Environmental and Geodetic Engineering, The Ohio State University, Columbus, OH, USA. Received 14 January 2016; revised 29 March 2016; accepted 3 April 2016; published online 17 May 2016 lysed cells, latent period) and outcome (e.g., lysis, lysogeny) of phage-host interactions, these have largely been studied only in a few phyla, for example, Actinobacteria, Firmicutes and $\gamma$-Proteobacteria, that under-represent environmental diversity (Holmfeldt et al., 2013). Further, even though lytic phages have variable efficiencies depending on host and environmental factors (You et al., 2002; Wang, 2006), infection data derives from common approaches, for example, plaque assays and spot tests, that favor investigating efficient (e.g., large fraction of infected/lysed cells and short latent periods) phage-host interactions (Dang and Sullivan, 2014).

In contrast, the lack of model systems to study inefficient infections (e.g., those with lower fraction of infected/lysed cells and long latent periods) is problematic, as natural ecosystems are dynamic and environmental conditions impact host diversity, density and/or physiology, as well as the efficiency of phage-host interactions (You et al., 2002; Abedon et al., 2003; Shao and Wang, 2008; Stocker, 2012; Mojica and Brussaard, 2014; Storms et al., 2014; Zeglin, 2015). To date, work on infection efficiencies is mostly theoretical (Abedon et al., 2001; You et al., 2002; 
Bragg and Chisholm, 2008) and there is a need for new phage- - host model systems, particularly those displaying inefficient infections and that are relevant in nature.

One such system is podovirus $\phi 38: 1$ infecting Cellulophaga baltica (Holmfeldt et al., 2014; Dang et al., 2015) of the Bacteroidetes phylum. This host phylum is abundant in the human gut and the oceans (Kirchman, 2002; Gomez-Pereira et al., 2010), and the phage is the only cultured representative for one of the four globally abundant virus types in the oceans (Roux et al., 2015a). Podovirus $\phi 38: 1$ is also well characterized as one of a collection of Cellulophaga baltica phages that have been examined using genomics, structural proteomics, quantitative host-range assays and infection dynamics (Holmfeldt et al., 2014; Dang et al., 2015). Such analyses revealed that $\phi 38: 1$ has a broad host range as it infects 13 of the 21 isolated C. baltica strains, contrasting other phages from the same collection, which have a narrower host range given that they can only infect 1-2 strains (Holmfeldt et al., 2014). Two of these 13 infected host strains, NN016038 and no. 18, are nearly identical genetically, as they display identical 16S-rRNA gene sequences (Holmfeldt et al., 2007) and share 93\% of their genes with average nucleotide identity of $>99.99 \%$ (this study). As well, such strains are physiologically nearly identical, as they have the same growth, size and morphology (Dang et al., 2015). Despite the large similarities between the host strains, $\phi 38: 1$ infection differs markedly as follows. Infection of the original host used for isolation (strain NN016038) is fast and efficient, as $\sim 60 \%$ of the cells are infected and lysed within $70 \mathrm{~min}$ (Dang et al., 2015). Contrastingly, infection of alternative host strain no. 18 under identical experimental conditions (i.e., same phage stock and titre, growth and infection parameters) is inefficient given its reduced adsorption, fewer $(\sim 30 \%)$ infected cells and a much longer latent period and cell lysis $(\sim 11 \mathrm{~h}$ latent period by plaque assay or $150 \mathrm{~min}$ by phageFISH) (Dang et al., 2015). Beyond the inefficient adsorption, the mechanisms driving intracellular inefficiency of infection, if any, are unknown.

Here we complemented these prior investigations to characterize the intracellular infection dynamics of variably efficient phage-host interactions by temporally quantifying DNA (via quantitiatve PCR (qPCR)), RNA (via RNA-sequencing (RNA-seq)) and phage particles (via electron microscopy (EM)) throughout $\phi 38: 1$ infection of each $C$. baltica host. These findings advance understanding of how viral infection efficiency varies across nearly identical bacterial hosts and are foundational for unveiling mechanisms of phage-host interactions in nature.

\section{Materials and methods}

Data availability

Reads from RNA-seq data are available at the Sequence Read Archive under the study number
SRP066570. All protocols can be found at https:// u.osu.edu/viruslab/protocols/. Scripts are in https:// bitbucket.org/MAVERICLab/rnaseq-cba38-1-phagehost-time-series as well as in http://mirrors.iplantcol laborative.org/browse/iplant/home/shared/iVirus/ Cellulophaga_Transcriptomics, where additionally the Supplementary Data set S1 can be found. The latter contains all statistical and functional analyses for the RNA-seq data, phage and bacterial genome analyses and the qPCR and transmission electron microscopy analyses.

\section{Phage-host manipulations}

Growth and infections of $C$. baltica host strains NN016038 (original) and no. 18 (alternative) with $\phi 38: 1$ were conducted as described previously (Holmfeldt et al., 2014; Dang et al., 2015). Briefly, cells were grown in Marine Luria Bertani medium to a density of $10^{8}$ cells per ml in early logarithmic stage. They were then infected with $\phi 38: 1$ in $50 \mathrm{ml}$ at a multiplicity of infection of 1.1 and 6.1 for N016038 and no. 18, respectively, which maximized the fraction of infected cells $(75 \%$ and $38 \%$ for the original and alternative host infections, respectively) as determined previously (Dang et al., 2015). Such differences were not problematic or likely to drive the differences in infection efficiency, as a previously determined wide span of multiplicity of infections (e.g., 0.1, 3,6) resulted in similar infection dynamics (Holmfeldt et al., 2014; Dang et al., 2015). Included were three infections and three no-phage controls where Marine Sodium Magnesium buffer was added instead of phage. After $15 \mathrm{~min}$ of phage-host adsorption time, replicates were diluted 10 -fold in Marine Luria Bertani and time 0 ('0 min') was established. Phage and cell abundance (via viable cell plating) were enumerated as described previously (Holmfeldt et al., 2014).

\section{Whole-genome comparison of both hosts}

Genomes from C. baltica NN016038 and no. 18 were compared through the online tool http://enve-omics. ce.gatech.edu/ani/http://enve-omics.ce.gatech.edu/ ani/ to obtain their average nucleotide identity. Genomes were further compared with Blastp (threshold of 50 on bit score, 0.001 on e-value and $99 \%$ of amino-acid identity of BLAST hit). For each genome, a protein not detected in the other was considered as 'unique' to this host. Genes unique to each host were surveyed for the presence of phage defense genetic features, including CRISPR genes, restriction modification, abortive infection mechanisms and toxin/antitoxin genes (Samson et al., 2013), as well as bacteriophage exclusion genes (Goldfarb et al., 2015) (Supplementary Dataset S1, tab GenomicDifferencesHosts and Supplementary Table S1). 
Quantitative PCR

Samples for qPCR were collected $(1 \mathrm{ml})$ for the original host (at 0, 15, 30, 45, 60, 75 and $90 \mathrm{~min}$ ) and the alternative host (at 0, 15, 30, 45, 60, 120 and $150 \mathrm{~min}$ ), centrifuged at $20817 \mathrm{~g}$ for $5 \mathrm{~min}$, the supernatant removed and pellets were stored at $-80^{\circ} \mathrm{C}$ until processing. Phage gene gp021, which was previously used for phage detection throughout infection (Holmfeldt et al., 2014), and bacterial housekeeping genes M667_14370 (C. baltica N016038) and M666_05390 (C. baltica no. 18), which have high, constant expression over time (i.e., edgeR false discovery rate $>0.05$; Supplementary Dataset S1, tabs RNASeq_original_host and RNASeq_alternative_host), were chosen as targets to be amplified by qPCR using the QuantiTect SYBR Green PCR Kit (Qiagen, Valencia, CA, USA; cat. no. 204145). Results are found in the Supplementary Data set S1 tab $q P C R$.

\section{Transmission electron microscopy}

Samples $(10 \mathrm{ml})$ were collected throughout each infection at 0, 45, 60 and $75 \mathrm{~min}$ (original host, $C$. baltica NN016038 infection) and at 0, 90, 120, 150 and $240 \mathrm{~min}$ (alternative host, C. baltica no. 18 infection), centrifuged for $10 \mathrm{~min}$ at $10000 \mathrm{~g}$ to remove the supernatant and then the pellet was fixed onto grids (200 mesh copper grids with carbonstabilized formvar support; Ted Pella, Redding, CA, USA), which were analyzed as described previously (Brum et al., 2005), to determine the frequency of visibly infected cells using a transmission electron microscope (CM12, Philips, Eindhoven, The Netherlands) to count the phage particles within cells. Complete protocols are available at http://u.osu. edu/viruslab/protocols/\#TransmissionElectronMi croscopy and raw data in the Supplementary Data set $\mathrm{S} 1$, tab TEM.

\section{RNA extractions}

Samples for RNA were collected (1-2 ml) from minutes 0, 15, 30, 45, 60 and 120 (original host) and from minutes 0, 20, 40, 60 and 120 (alternative host), and spun down at $20817 \mathrm{~g}$ for $5 \mathrm{~min}$. The supernatant was then removed and tubes were flashfrozen in liquid nitrogen and stored at $-80^{\circ} \mathrm{C}$ until the extraction. RNA extractions were performed with the RNeasy Mini Kit (Qiagen; cat. no. 74104), DNase treated with TURBO DNA-free (Ambion, Carlsbad, CA, USA; AM1907) and concentrated to $20 \mu l$ with the RNA Clean and Concentrator-5 Kit (Zymo Research Corporation, Irvine, CA, USA; R1015). The RNA integrity number and concentration of the extracted RNA was determined via the Agilent Bioanalyzer 2100 (Agilent Technologies, Santa Clara, CA, USA). For all samples, nomenclature includes the time followed by infection (I) or control (C) and the replicate (R) number. For example, biological replicate 1 of the infection at $15 \mathrm{~min}$ is ' $15 \mathrm{~min}$ I R1'.
RNA-seq, read mapping to host and phage genomes and coverage calculations

Libraries of complementary DNA were prepared for SOLiD 5500 XL (Applied Biosystems, Foster City, CA, USA) sequencing according to the manufacturer's protocol (Life Technologies, Carlsbad, CA, USA). All sequencing data is available at the Sequence Read Archive under study number SRP066570. The 50 bp reads were mapped to the genomes of the corresponding host, $C$. baltica NN016038 (GenBank no. CP009887.1) or C. baltica \#18 (GenBank \# CP009976), and phage $\$ 38: 1$ (GenBank no. NC_021796.1) using Bowtie2 v.2.14. Coverage of the phage and host genomes was calculated as the number of base pairs mapped to a genome divided by the genome length (Supplementary Tables S2).

Phage genome reorganization and annotation

The publicly available phage genome (GenBank no. NC_021796.1) was reordered to accommodate the transcriptional pattern, that is, by making the first gene represented coincide with the first gene transcribed and can be found at http://mirrors. iplantcollaborative.org/browse/iplant/home/shared/ iVirus/Cellulophaga_Transcriptomics. New predicted functional annotations were incorporated by comparing unknown gene products to publicly available protein sequences via Blastx, Blastn, BlastP, InterProScan and NCBI Conserved Domain and overlapping results were selected as an approximation of the protein's function, which was designated as 'putative'.

Statistical analyses of the phage and host transcriptomes

Scripts for the following analyses are provided at https://bitbucket.org/MAVERICLab/rnaseq-cba381-phage-host-time-series.

Values for $\log _{2}$ RPKM (reads per kilobase of transcript per million mapped reads) and results from differential expression (DE) analyses for all phage and both host genes can be found in the Supplementary Dataset S1, tabs RNASeq_original_host, RNASeq_phage_on_OriginalHost, RNA Seq_alternative_ host, RNASeq_phage_on_AlternativeHost, RNASeq OriginalHostCategories, RNASeqAlternativeHost Categories, RNASeqOriginalHostPhageDefense and RNA SeqAlternHostPhageDefense.

Normalization of the read counts, calculation of RPKM and heat map representation. Read counts of phage and host were normalized separately using the $\mathrm{R}$ software package edgeR in Bioconductor (Robinson et al., 2010). The resulting matrices were used for calculating the RPKM values (Mortazavi 
et al., 2008) for all genes and for DE analyses (see below).

Sample clustering. After normalization and calculation of $\log _{2}$ RPKM, host-infected and control samples were hierarchically clustered via Pearson's correlation using the pvclust package (Suzuki and Shimodaira, 2006) in R. Node support was calculated through multiscale resampling (10 000 bootstraps; Supplementary Figure S3). From the original host infection, samples ' 0 min I R3', ' 15 min I R3', '30 min I R3' and ' 120 min C R3' were discarded owing to the lack of clustering with samples from the same time point and type (Supplementary Figure S3a).

DE analyses. DE was calculated between (i) infected and control host samples at every time point, (ii) between time points of host control samples and (iii) between time points of phageinfected samples. Statistical package edgeR (Robinson et al., 2010) was used and genes with a false discovery rate $<0.05$ were considered as DE. Further, the genes obtained as DE within time points (e.g., 0 min vs the others) in host control samples were removed from the list of genes differentially expressed between infected and control. Although there were none in the original host, there were $13(0.3 \%$ of total genes; Table 2$)$ in the alternative host. Fold change (in $\log _{2} \mathrm{FC}$ ) for those differentially expressed genes was calculated as the difference in expression (i.e., $\log _{2}$ RPKM) between infected and control. To compare the two infections, the RPKM obtained from infected samples was assumed to derive from the sum of infected and uninfected cells such that $\mathrm{RPKM}_{\mathrm{i}}=a \times \mathrm{RPKM}_{\mathrm{x}}+$ $(1-a) \times \mathrm{RPKM}_{\mathrm{c}}$, where $\mathrm{RPKM}_{\mathrm{i}}$ includes the total RNA-seq signal obtained from 'infected' samples (which includes infected and uninfected cells), $\mathrm{RPKM}_{x}$ is the expression of just the infected cells (and what needs to be solved for in the previous equation), $a$ is the fraction of infected cells and $\mathrm{RPKM}_{c}$ is the expression of non-infected (control) cells. The resulting normalized $\log _{2} \mathrm{FC}$ equaled $\log _{2} R P K M_{x}-\log _{2} R P K M_{c}$. Genes were overexpressed if normalized $\log _{2} \mathrm{FC}>0$ and underexpressed if normalized $\log _{2} \mathrm{FC}<0$.

Temporal expression clusters. Phage genes from infected samples were clustered according to their temporal expression profile (based on $\log _{2} \mathrm{RPKM}$ ). All time points were compared against each other using edgeR (see above), and the resulting genes with a false discovery rate $<0.05$ were hierarchically clustered to determine the number of stable clusters as described previously (Lindell et al., 2007). Briefly, the scaled $\log _{2}$ RPKM data set was hierarchically clustered using Pearson's correlation and resampled using the clusterStab $\mathrm{R}$ package (Smolkin and Ghosh, 2003).

Functional group assignment to differentially expressed host genes

Host genes found DE between infected and control samples at every time point (see above) were placed into one of 14 functional categories based on MG-RAST (Meyer et al., 2008) (i.e., amino-acid metabolism, cell cycle and division, cell signaling, cell wall remodeling, DNA metabolism, energy, motility, nucleotide metabolism, prophages and transposons, transport, protein metabolism, RNA metabolism, stress, unknown) by grouping similar functions into the same category using R (Figure 4 and Supplementary Dataset S1, tabs RNASeqOriginalHostCategories and RNASeqAlternativeHostCategories).

\section{Codon adaptation index}

The codon usage table for the phage and both the original and the alternative hosts as well as the codon adaptation index) (Sharp and Li, 1987) were calculated with the EMBOSS software (Rice et al., 2000).

\section{Results and discussion}

RNA-seq of phage $\$ 38: 1$ and two similar C. baltica host strains with contrasting lytic infections

Phage $\phi 38: 1$ (a $70 \mathrm{~kb}$ double-stranded DNA podovirus ) was previously shown to differentially infect two nearly identical $C$. baltica host strains, NN016038 (original) and no. 18 (alternative), which could be at least partially explained by differential adsorption efficiencies (Dang et al., 2015). Here we assessed whether other features of the phage or host genomes or transcriptomes might also contribute to variability in infection efficiency across these hosts.

Genomically, both host strains are $100 \%$ identical at the 16S rRNA level (Holmfeldt et al., 2007) and share $93 \%$ of their protein-coding genes with an average nucleotide identity of $99.99 \%$ (see Materials and methods). Among the unique genes in each host, no intact phage defense mechanisms were observed when surveyed for CRISPR, complete bacteriophage exclusion cassettes, restriction modification, abortive infection or toxin/antitoxin genes (Supplementary Table S1, tab GenomicDifferencesHosts) (Samson et al., 2013; Goldfarb et al., 2015).

Beyond adsorption and common defense mechanisms, differences in expression of phage or host genes could be involved in determining differences in infection efficiency. To assess this hypothesis, gene expression of phage and the $C$. baltica hosts were temporally quantified (Dang et al., 2015) via time-resolved, whole-genome transcriptome sequencing (Figure 1). For each phage-host pair, three infections and no-phage controls were diluted 10-fold to minimize secondary infections after $15 \mathrm{~min}$ 
a Original host (C. baltica NN016038) infection

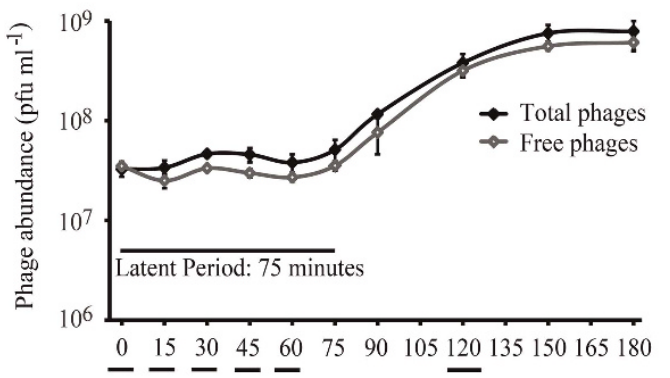

Time (minutes)

C

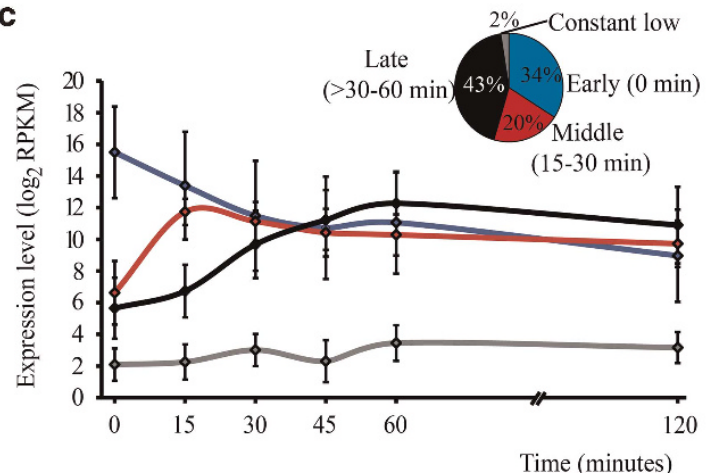

b

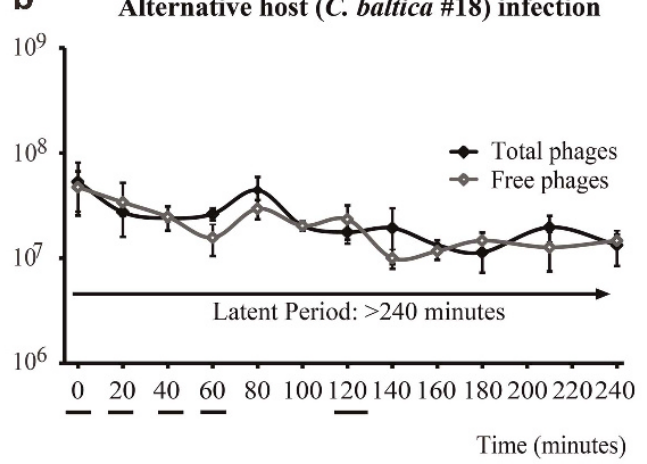

d

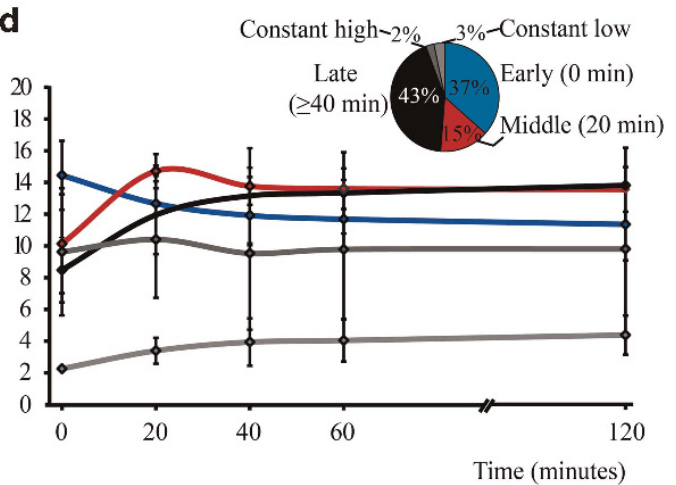

Figure 1 Infection dynamics of phage $\$ 38: 1$ on its two C. baltica host strains. (a) Efficiently lytic infection on the original host displays a 75 min infection cycle via one-step growth curve, whereas (b) the inefficiently lytic infection shows no significant phage production over 4 h. (c) The efficiently lytic phage expresses genes in early, middle, late and constant low temporal groups, similar to when infecting the alternative host (d), where genes are also expressed in constant high temporal groups. Represented are the average and standard error of the biological triplicates (a and $\mathbf{b}$ ) or the genes in each group (c and $\mathbf{d})$. PFU, plaque forming units. Underlined are the time points assayed in the whole-genome transcriptional analyses.

of phage-host adsorption when time ' 0 ' was established (see Materials and methods). The observed infection dynamics were consistent with the previous work (Dang et al., 2015), with 75\% infected cells and a $75 \mathrm{~min}$ latent period (two-tailed $t$-test, $P<0.05$; Figure 1a) on the original host, and $38 \%$ infected cells with no significant change in phage abundance over $4 \mathrm{~h}$ on the alternative host (two-tailed $t$-test, $P>0.05$; see Materials and methods and Figure 1b).

Transcriptome data were obtained via Sequencing by Oligonucleotide Ligation and Detection (SOLiD; see Materials and methods), generating $1.7 \times 10^{7}-$ $1.4 \times 10^{8}$ and $1.6 \times 10^{7}-1.1 \times 10^{8}$ reads per sample in the original and alternative host infections, respectively, which covered both genomes in each experiment $\geqslant 40$ times on average (Supplementary Tables S2 and Supplementary Figure S1). Strand-specific sequencing provided expression of the phage intergenic regions, which enabled identification of six putative phage non-coding RNAs (Supplementary Figure S2 and Supplementary Table S4), which are now routinely discovered in phage transcriptome sequencing experiments and commonly have gene regulatory functions (Doron et al., 2015; Lin et al., 2015). Phage and host transcriptomes were further analyzed to explore the biology that drives efficient vs inefficient phage-host interactions as described in the following sections.

\section{Universalities of lytic phage infection}

The phage transcriptome during the efficiently lytic infection on the original host. The phage transcriptome when infecting the original host revealed that most phage genes were expressed in three temporally regulated groups-early (34\% of total genes), middle $(20.3 \%$ of total genes) and late $(43.1 \%$ of total genes)-and a few genes with constant temporal expression-constant low (2.4\% of total genes) (Figure 1c and Table 1). These gene groups were largely consecutive to each other and physically arranged such that early, middle and late progressed from one end to another in the phage genome (Figure 2). Exceptions to these patterns in $\phi 38: 1$ included three genes with unknown functions that were expressed as middle and located within the late genes (Figure 2 and Supplementary Table S4), presumably prolonging expression or degradation of the transcripts until late infection, as posited for other phage-host systems (Lindell et al., 2007; Pavlova et al., 2012; Ceyssens et al., 2014).

Functional gene annotations provided insight into how $\phi 38: 1$ phage infection progresses throughout an efficient lytic infection (Figure 2 and Supplementary Table S4). Early genes functioned in take-over to express phage genes and control host metabolism 
Table 1 Summary statistics of phage $\phi 38: 1$ 's transcriptome during the infection on C. baltica original (NN016038) and alternative (no. 18) host strains $($ FDR $<0.05)$

ф38:1 on C. baltica NN016038

Number of genes \% of total genes Highest expression $\phi 38: 1$ on C. baltica no. 18

Number of genes \% of total genes Highest expression

\begin{tabular}{|c|c|c|c|c|c|c|}
\hline Early & 42 & 34.2 & $0.0-15 \min$ & 45 & 36.6 & $0 \mathrm{~min}$ \\
\hline Middle & 25 & 20.3 & $15-30 \mathrm{~min}$ & 18 & 14.6 & $20 \mathrm{~min}$ \\
\hline Late & 53 & 43.1 & $45-60 \mathrm{~min}$ & 53 & 43.1 & $>40 \mathrm{~min}$ \\
\hline Constant low & 3 & 2.4 & - & 4 & 3.3 & - \\
\hline Constant high & - & - & - & 3 & 2.4 & - \\
\hline
\end{tabular}

Abbreviations: C. baltica, Cellulophaga baltica; FDR, false discovery rate.

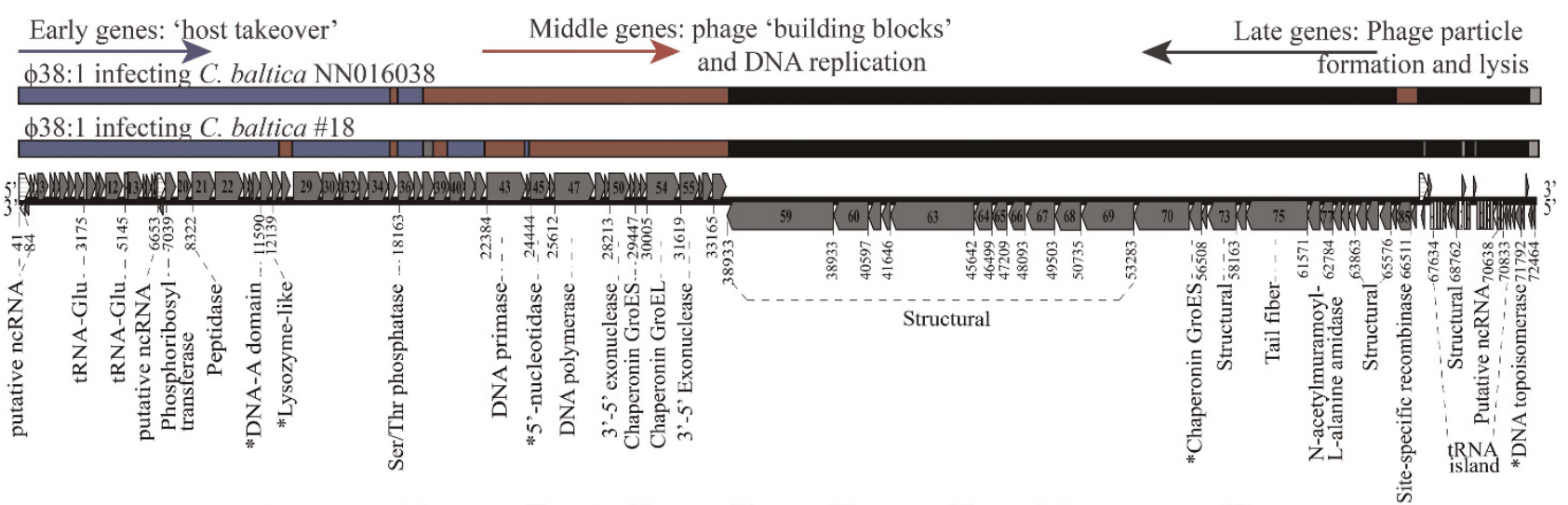

Legend: *Putative protein functions; 罗 ncRNA; $\because$ tRNA; $\square$ CDS; $\square$ Early; $\square$ Middle; $\square$ Late; $\square$ Constant high; $\square$ Constant low

Figure 2 Whole-genome transcriptome of phage $\phi 38: 1$ infecting its two $C$. baltica host strains. Genes in the plus strand (5'-3') are represented above the genome line (black) and genes in the minus strand $\left(3^{\prime}-5^{\prime}\right)$ are below the line. Some numbers inside the genes, representing gene product ( $\mathrm{gp}$ ) identifiers, as well as their function are displayed for guidance. Complete information can be found in the Supplementary Table S4 and Supplementary Dataset S1.

(e.g., ribosyltransferase gp079; Serine/Threonine phosphatase gp036). Additionally, some of these genes (lysozyme (gp027) (Rossmann et al., 2004), an $\alpha$-mannosidase (gp030) (Prehm and Jann, 1976) and an S-layer-related protein (gp034) (Mann et al., 2005)) likely prepared the host cell to defend against superinfection via cell wall modifications. Alternatively, the lysozyme-like proteins may instead bind to RNA polymerase, as observed in coliphage T7 (Omcallister and Wu, 1978; Cheetham and Steitz, 2000), and function to benefit the phage via modulating host gene expression. As infection progressed, middle genes helped provide the phage 'building blocks' for phage reproduction, as the phage expressed genes that appeared to modulate nucleic acid and protein catabolism (e.g., exonuclease gp050; proteasome gp039, respectively), phage DNA replication (e.g., DNA primase gp043) and energy metabolism (e.g., phosphomannomutase gp041; NAD-dependent epimerase/dehydratase gp043). Finally, late genes promoted viral particle formation (e.g., major capsid protein gp067; tail fiber gp071) and cell lysis (e.g., $\mathrm{N}$-acetylmuramoyl-L-alanine amidase gp077; Figure 2 and Supplementary Table S4).

Taken together these patterns are consistent with the temporal regulation that is common among lytic phages. Particularly, these phages appear to strongly regulate gene expression via either two ('early' and 'late'; Loskutoff et al., 1973; Pene et al., 1973; Poranen et al., 2006; Fallico et al., 2011; Ainsworth et al., 2013) or three ('early', 'middle'/'intermediate' and 'late'; Lindell et al., 2007; Legendre et al., 2010; Pavlova et al., 2012; Lavigne et al., 2013; Ceyssens et al., 2014; Doron et al., 2015; Lin et al., 2015) groups of physically proximal genes with similar functions. Such functions are tailored to the progression of the infection, with early host takeover and phage genome replication followed by late particle formation and cell lysis. Such patterns have now been observed across a wide range of phage systems, from classic phages (e.g., lambda (Lobocka et al., 2004), T4 (Miller et al., 2003) and PRD1 (Poranen et al., 2006)) to pathogens (e.g., Pseudomonas phiLuz19 (Lavigne et al., 2013)) and environmental viruses (e.g., giant viruses like mimivirus (Legendre et al., 2010) and Pseudomonas phiKZ (Ceyssens et al., 2014)) or the marine cyanophages (PSSP7 (Lindell et al., 2007), Syn9 (Doron et al., 2015) and PSSM2 (Lin et al., 2015)). The characterization of these phages has revealed a relative universality in regulating phage gene expression during a lytic infection, regardless of the host and the environment.

Phage transcriptome on the alternative host is similar to that on the original host. While the 
alternative host infection dynamics was significantly delayed relative to that in the original host (Figure 1), the phage transcriptome was remarkably similar across infection of both hosts (Figures 1 and 2). First, phage genes were similarly spatiotemporally regulated as $36.6 \%, 14.6 \%$ and $43.1 \%$ of the total genes were expressed as early, middle and late, respectively, with the few remaining expressed constantly as constant high $(2.4 \%$ of total genes) or constant low (3.3\% of total genes) (Figures 1 and 2, Table 1 and Supplementary Table S4). Second, nearly all $(90.2 \%)$ of the phage genes were expressed in the same temporal categories observed when infecting the original host (Figure 2 and Supplementary Table S4). Only one of the remaining genes (gp085) could be functionally annotated, as a putative site-specific recombinase. Since $\phi 38: 1$ does not integrate its genome into the alternative host chromosome DNA (Holmfeldt et al., 2014), gp085 might function either as an integrase in another host or be involved in phage DNA replication, similar to other members of this gene family (Cox, 2001). Third, phage gene expression was nearly identical across both hosts by the end of the efficiently lytic infection (i.e., $60 \mathrm{~min}$; Figure 1). Thus, even though expression of genes such as gp085 was delayed in the alternative host compared with the original host, potentially contributing to the inefficient infection, the overall similarities in genome-wide phage expression when infecting each host suggests that the phage was unlikely to have driven the infection inefficiencies across these hosts.

Host transcriptomes drive infection efficiency While the phage transcriptomes were similar between infections, the host transcriptomes were strikingly different. First, relative to the uninfected controls, early host gene expression was globally underexpressed in the original host relative to the alternative host (Figure 3). The former suggests that the phage successfully redirected original host metabolic machinery towards making phage progeny, and is consistent with observations in some coliphage and cyanophage systems (Koerner and Snustad, 1979; Lindell et al., 2007; Fallico et al., 2011; Doron et al., 2015). Second, fewer genes were differentially expressed at each time point in the original $(0.7-6.3 \%)$ vs the alternative $(6-12 \%)$ host, with more diversity of genes differentially expressed in the alternative host $(22.1 \%)$ than in the original (9.4\%) host (Table 2 and Supplementary Figure S4). Small host responses to lytic phage infection, such as those observed here for the original host, are common among other genome-wide transcriptome studies of lytic model systems (Poranen et al., 2006; Ravantti et al., 2008; Fallico et al., 2011; Ainsworth et al., 2013; Lavigne et al., 2013; Ceyssens et al., 2014) and suggest a fast host takeover by the phage. However, the larger transcriptional response to phage infection observed in the alternative host is

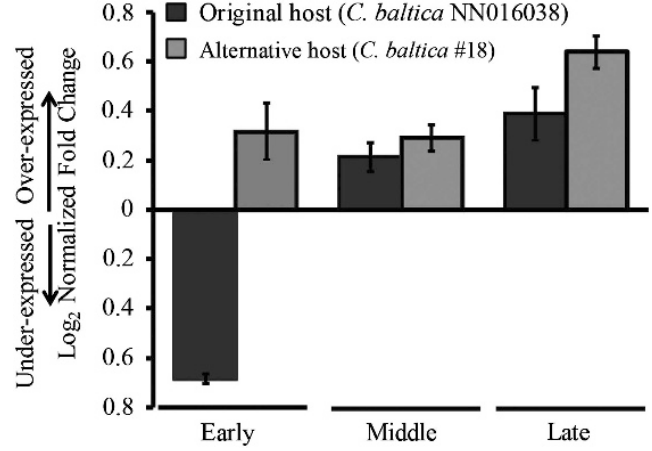

Figure 3 Transcriptional response of $C$. baltica to phage $\phi 38: 1$ infection. Plotted is the average expression of all differentially expressed genes during infection relative to control (fold change), normalized by fraction of infected cells. Error bars represent the standard deviation of the biological replicates.

uncommon and comparable only to a lytic cyanophage that strongly depends upon its host's metabolic machinery (Lindell et al., 2007). Thus, either $\phi 38: 1$ is more dependent on the host's metabolic machinery in the alternative host or this host more strongly defends against phage infection.

Functional analyses of differentially expressed host genes (Figure 4) helped discriminate between those two hypotheses, specifically suggesting benefits to phage reproduction in the original but not in the alternative host, as follows. First, the fold change of expression between infected and control was larger by $60 \mathrm{~min}$ (end of infection) in the original host than by $120 \mathrm{~min}$ in the alternative host (Figure 4), suggesting that the genes differentially expressed in the alternative host were not expressed at optimal levels as in the original host. Second, each stage of phage infection (i.e., early, middle and late) seemed supported by beneficial functions in the original host, but not in the alternative host (Figure 4 and Supplementary Dataset S1, tabs RNASeqOriginalHostCategories, RNASeqAlternativeHostCategories). For example, during early infection, the original host overexpressed genes involved in RNA, nucleotide and amino-acid metabolism, whereas the alternative host overexpressed a tRNAPro gene (protein metabolism category). This tRNAPro gene was expressed in the original host, but not differentially expressed during phage infection, which suggests that the gene product is not critical for the efficient infection. Most underexpressed genes in the original host were involved in the stress response, likely indicating the phage shutting down host defenses against infection (Figure 4 and Supplementary Dataset S1, tabs RNASeqOriginalHostCategories, RNASeqAlternativeHostCategories). Consistent with this, no such genes were underexpressed in the alternative host, where infection was challenged by host defenses and the infection was inefficient.

During middle gene expression, the original host overexpressed RNA (e.g., RNAP gene), nucleotide 
Table 2 Summary statistics of the differentially expressed genes in C. baltica original (NN016038) and alternative (no.18) host strains $($ FDR $<0.05)$

C. baltica NN016038

\begin{tabular}{|c|c|c|c|c|c|c|c|c|c|c|c|c|c|c|c|}
\hline & \multicolumn{6}{|c|}{$I$ vs $C$} & \multirow{2}{*}{$\begin{array}{c}C \text { vs } C \\
A l l^{\mathrm{a}}\end{array}$} & \multirow{2}{*}{$\begin{array}{c}\text { Total } \\
D E^{\mathrm{a}}\end{array}$} & \multicolumn{5}{|c|}{$I$ vs $C$} & \multirow{2}{*}{$\begin{array}{c}C \text { vs } C \\
A l l^{\mathrm{a}}\end{array}$} & \multirow{2}{*}{$\begin{array}{l}\text { Total } \\
D E^{\mathrm{a}, \mathrm{b}}\end{array}$} \\
\hline & $0 \min$ & $15 \mathrm{~min}$ & $30 \mathrm{~min}$ & $45 \mathrm{~min}$ & $60 \mathrm{~min}$ & $120 \mathrm{~min}$ & & & $0 \min$ & $20 \mathrm{~min}$ & $40 \mathrm{~min}$ & $60 \mathrm{~min}$ & $120 \mathrm{~min}$ & & \\
\hline Genes & 26 & 238 & 117 & 105 & 94 & 127 & 0 & 354 & 252 & 267 & 356 & 229 & 456 & 13 & 844 \\
\hline$\%$ of total genes & 0.7 & 6.3 & 3.1 & 2.8 & 2.5 & 3.4 & 0.0 & 9.4 & 6.6 & 7.0 & 9.3 & 6.0 & 12.0 & 0.5 & 22.1 \\
\hline
\end{tabular}

Abbreviations: C, control; C. baltica, Cellulophaga baltica; DE, differential expression; FDR, false discovery rate; I, infected.

aNon-redundant genes DE throughout the infection.

${ }^{\mathrm{b}}$ After removing the genes DE in control samples.

a

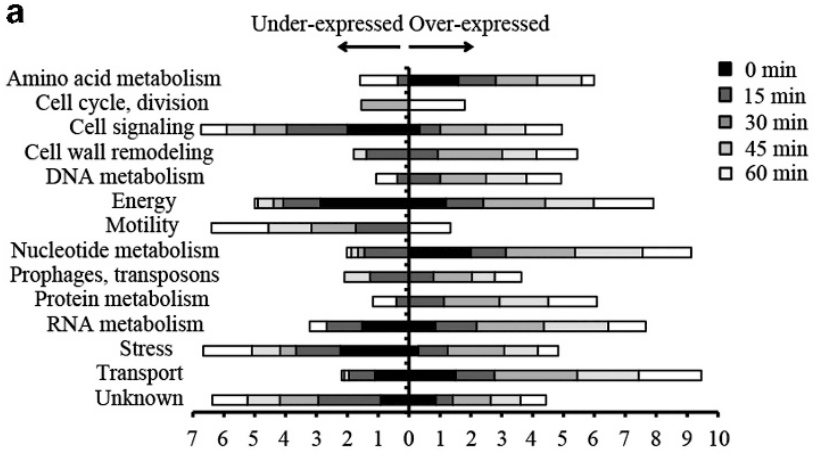

b

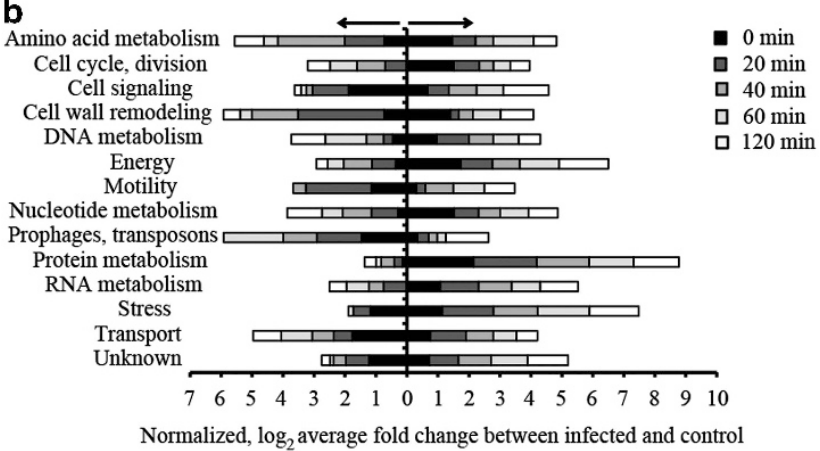

Figure 4 Host $C$. baltica's response to phage $\phi 38: 1$ infection. (a) Original (NN016038) and (b) alternative (no.18) host's transcriptional categories during infection, normalized to account for the fraction of infected cells. Detailed figures for DNA and protein metabolism are represented in Figure 5.

and DNA metabolism genes (Figure 4a), presumably to make phage transcripts and DNA (Brown and Bidle, 2014). Modulating the expression of RNAP in the original host was expected, as $\phi 38: 1$ does not encode its own RNAP, and thus must repurpose the host RNAP towards transcribing phage genes. In contrast, the alternative host did not overexpress any of these functions and instead most highly expressed the tRNA-Asp gene again (which was not differentially expressed in the original host) and stress response genes (Figure $4 \mathrm{~b}$ and Supplementary Dataset S1, tabs RNASeqOriginalHostCategories, RNASeqAlternativeHostCategories). Among underexpressed genes, the largest effect was in motility in both hosts and cell wall biosynthesis in the alternative host, suggesting the phage seeks to mitigate the host's defenses against infection or prevent unnecessary energy consumption in processes not beneficial to phage infection.

Finally, host transcriptomes during late infection again revealed cellular environments responding differently in the efficient vs inefficient infections. The original host overexpressed transport and energy genes (Figure 4a), perhaps reflecting the energetic demand of the lysis process that would follow. In addition, underexpression of stress genes suggested a phage-derived need to prevent host metabolic shutdown (Brissette et al., 1990) before phage-controlled cell lysis, as in coliphage M13 (Karlsson et al., 2005). In contrast, the alternative host overexpressed genes involved in stress and protein metabolism (tRNA-Asp) genes (at $60 \mathrm{~min}$ ) while underexpressing amino-acid metabolism genes (Figure 4b), none of which aligned with the transcriptional response observed in the efficient infection. Additionally, the recurring DE of tRNA genes in the alternative host was not driven by any obvious codon bias across the hosts (Supplementary Figure S5), which is consistent with their nearcomplete lack of variation in $\% \mathrm{G}+\mathrm{C}(34.7 \%$ and $38.1 \%$ in both hosts and the phage, respectively), and suggests that such transcriptional pattern is unlikely to explain the infection differences between the hosts.

Inefficient infections are driven by host defenses against phage DNA and protein

At this point, it appeared that inefficient phage infection was driven, not by differential phage gene expression, but by host defenses throughout the infection against phage. To further test this hypothesis we sought to evaluate possible host responses against phage beyond transcription, such as DNA and protein production (Figure 5), that could be differentially transcribed in the efficient vs inefficient infections. First, investigation of core host genes potentially involved in phage defense (e.g., CRISPRs, restriction-modification, bacteriophage exclusion, abortive infection and toxin/antitoxin; 
Goldfarb et al., 2015; Samson et al., 2013) based on their annotation suggested the presence of methyltransferases and endonucleases that could be part of a restriction-modification system (Supplementary Table S1, tabs RNASeqOriginalHostPhageDefense and RNASeqAlternHostPhageDefense) with only one gene (endonuclease M666_14090) differentially expressed in the alternative host (Supplementary Table S1, tab RNASeqAlternHostPhageDefense). This suggested differential regulation of phage DNA production in the alternative host, previously supported by phageFISH data, indicating that phage DNA replication occurred in both hosts, but took nearly twice as long in the alternative host (Dang et al., 2015). Second, following this lead, transcriptomics revealed that the original host overexpressed DNA degradation genes (e.g., nucleases) by middle infection (Figure 5a), which was coincident with a $63 \%$ reduction (range: $15-90 \%$ ) of host relative to phage DNA from middle to late infection (Figure 5b). This suggested that the original host DNA was efficiently degraded, presumably to enable phage DNA replication given that (i) degraded host DNA can be recycled for phage DNA replication (Koerner and Snustad, 1979; Lavigne et al., 2013), and (ii) the phage expressed genes for replicating its genome (Figure 2) as phage DNA increased and host DNA decreased (Figure 5b). Here, the alternative host also overexpressed its DNA degradation genes early, and then underexpressed them late (including endonuclease M666_14090) (Figure 5a). However, phage DNA in the alternative host increased late instead of early as it did in the original host, was $>50 \%$ less abundant and plateaued later (by $60 \mathrm{~min}$ ) as compared with the original host (Figure 5b). These findings suggest that the alternative host contributed to the inefficient infection by specifically reducing and delaying phage DNA production.

Third, the expression pattern of host protein translation genes suggested that the alternative host defended against phage protein production as well, and experimental measurements following the formation of viral particles confirmed this. Specifically, the original host over-expressed translation genes from middle to late infection (Figure 5c), at which time phage particles were increasingly detected by EM (per cell averages of $\geqslant 9.4$ particles at $60-75 \mathrm{~min}$; Figure 5d). In contrast, throughout the inefficient infection, the alternative host under-expressed translation genes (Figure 5c), and phage particle abundances were delayed (by $>30 \mathrm{~min}$ ) and reduced (by $>60 \%$ ) as detected by EM (per cell averages of 3.5 particles at $90 \mathrm{~min}$; Figure $5 \mathrm{~d}$ ). As all viruses are thought to depend on host translational machinery to make viral particles (Walsh and Mohr, 2011), the timing of over-expressed translation genes in the original host coinciding with the formation of phage particles presumably was designed to help make such particles, whereas the failure to be overexpressed in the alternative host contributed to yet another inefficiency of that infection.

An emerging paradigm of the biology of phage infection Taken together, these data and previous findings (Holmfeldt et al., 2014) suggest that phage $\phi 38: 1$ infection inefficiency is multidimensional as it derives initially from reduced phage adsorption to
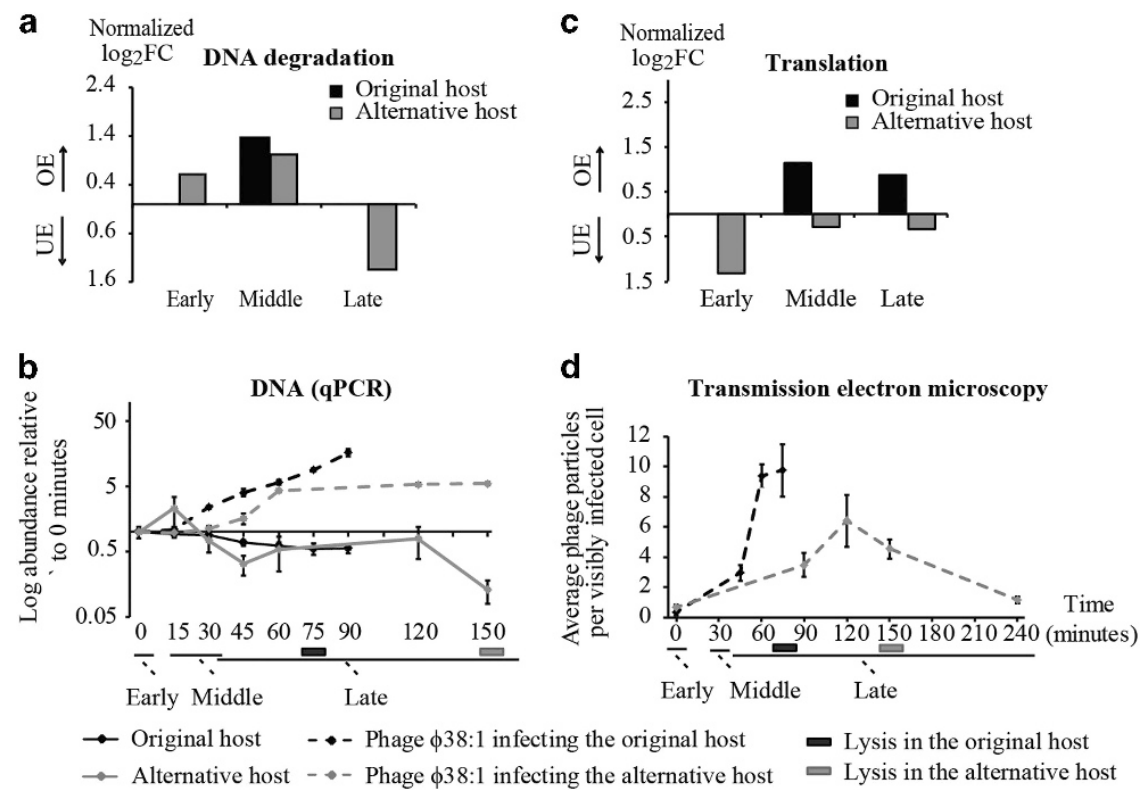

Figure 5 Regulation of the efficient and inefficient C. baltica phage-host interactions. Dynamics of (a and b) DNA replication and of (c and d) protein production in the original and alternative C. baltica hosts' infection with phage $\phi 38: 1$. Gene expression is represented as the difference between infected and control (fold change, FC), normalized by the different fraction of infected cells in each host (normalized $\log _{2} \mathrm{FC}$ ), resulting in overexpression (OE) or underexpression (UE). qPCR data is represented relative to time 0 min. For all analyses, the average of three biological replicates and their standard error are displayed. 
the alternative host, but then intracellularly from failure to shut down host defenses in contrast to the original host. This likely leads to activating a stress response in the alternative host throughout middle and late stages of infection that is not observed in the original host, where stress genes are instead underexpressed early (Supplementary Figure S6). Consequently, throughout infection, such defenses in the alternative host succeeded at delaying synthesis of new phage genomes and virions. This complements current knowledge of bacterial defense mechanisms against viral infections, largely focused at early stages of infection, where phage attack is stymied through cell surface receptor modification and various ways of phage DNA degradation (Samson et al., 2013). Instead, in our system, host defenses do not eradicate the virus; rather, they collectively and persistently lead to an inefficient infection, impacting phage adsorption, DNA and protein production.

As sequencing costs drop and non-traditional model systems are more routinely explored, knowledge of the intracellular dynamics of phage-host interaction are coming to light. For example, recent findings in a marine cyanophage infecting a broad range of hosts also showed similar phage transcription across the hosts and that host expression drove the differences between infections (Doron et al., 2015). Taken together, with our findings, this places new emphasis on the role of the host cellular environment (measured here though the transcriptome) in enabling phage infection across diverse hosts. That phage gene expression is similar regardless of the host genetic background might also represent an emerging paradigm as it has now been observed in phage-bacterial systems ranging from heterotrophs to cyanobacteria. Additionally, our experimentally supported functional analyses in an environmentally relevant Bacteroidetes virus provide a mechanistic foundation for better understanding the efficiency of viral infections across hosts.

\section{Conclusions}

Phage-host interactions are fundamental for understanding microbial ecosystems, yet research to date has largely ignored the inefficient phage-host interactions that are likely common in the environment. The model system investigated here represents one of the most abundant phage types in the global oceans (Roux et al., 2015a) and has a previously characterized broad host range (Holmfeldt et al., 2014) with which to analyze intraspecies phage-host interactions, as well as how one phage can differentially infect two nearly identical host strains. As knowledge of bacterial and viral diversity, abundance and ecological relevance advances (e.g., Rinke et al., 2013; Roux et al., 2015b), it becomes critical to expand mechanistic understanding of virus-host interaction types beyond those displayed by traditional model systems. Developing environmental model systems has been experimentally challenging owing to slow growth and a lack of background knowledge and genetic tools. However, emerging technologies, such as genome-wide transcriptomics, offer new and powerful windows into the mechanistic underpinnings of environmentally relevant virus-host interactions. Taken together with new approaches to quantitatively survey viral diversity (Duhaime and Sullivan, 2012; Solonenko et al., 2013) and virus-host interactions in the environment (reviewed in Dang and Sullivan, 2014), the field is poised to develop the foundational knowledge critical to model and understand the ecological impacts of virus-host interactions (Weitz et al., 2015), as well as guide efforts to manipulate infections that will improve bacterial-based biotechnological productions and human health (Rohwer and Segall, 2015).

\section{Conflict of Interest}

The authors declare no conflict of interest.

\section{Acknowledgements}

We thank members from the Tucson Marine Phage Lab at the University of Arizona, especially Lacey Orsini, Sarah Schwenck, Clayton Pierce, Cassidy Danbury and Mario Moreno for technical support; Dr W Day from the Arizona Health Sciences Center Imaging Core facility for TEM support; and Dr H Mitchell at PNNL for initial bioinformatics support. Partial support for CHV came from the NIH Graduate Training Grant in Biochemistry and Molecular Biology T32 GM008659. SR was partly supported by a grant to the UA Ecosystem Genomics Institute through the UA Technology and Research Initiative Fund and the Water, Environmental, and Energy Solutions Initiative. Part of the research was performed using the Environmental Molecular Sciences Laboratory (EMSL), a national scientific user facility sponsored by the Department of Energy's Office of Biological and Environmental Research and located at PNNL. This work was also funded by the Gordon and Betty Moore Foundation grants (GBMF no. 2631, 3790) and a DOE EMSL User Award (no. 47930) to MBS.

\section{References}

Abedon ST, Herschler TD, Stopar D. (2001). Bacteriophage latent-period evolution as a response to resource availability. Appl Environ Microbiol 67: 4233-4241.

Abedon ST, Hyman P, Thomas C. (2003). Experimental examination of bacteriophage latent-period evolution as a response to bacterial availability. Appl Environ Microbiol 69: 7499-7506.

Ainsworth S, Zomer A, Mahony J, Van Sinderen D. (2013). Lytic infection of Lactococcus lactis by bacteriophages Tuc2009 and c2 triggers alternative transcriptional host responses. Appl Environ Microbiol 79: 4786-4798. 
Bragg JG, Chisholm SW. (2008). Modeling the fitness consequences of a cyanophage-encoded photosynthesis gene. PLoS One 3: e3550.

Brissette JL, Russel M, Weiner L, Model P. (1990). Phage shock protein, a stress protein of Escherichia coli. Proc Natl Acad Sci USA 87: 862-866.

Brown CM, Bidle KD. (2014). Attenuation of virus production at high multiplicities of infection in Aureococcus anophagefferens. Virology 466-467: 71-81.

Brum JR, Steward GF, Jiang SC, Jellison R. (2005). Spatial and temporal variability of prokaryotes, viruses, and viral infections of prokaryotes in an alkaline, hypersaline lake. Aquatic Microbial Ecol 41: 247-260.

Ceyssens PJ, Minakhin L, Van Den Bossche A, Yakunina M, Klimuk E, Blasdel B et al. (2014). Development of giant bacteriophage varphiKZ is independent of the host transcription apparatus. J Virol 88: 10501-10510.

Cheetham GM, Steitz TA. (2000). Insights into transcription: structure and function of single-subunit DNAdependent RNA polymerases. Curr Opin Struct Biol 10: $117-123$.

Cox MM. (2001). Historical overview: searching for replication help in all of the rec places. Proc Natl Acad Sci USA 98: 8173-8180.

Dang VT, Howard-Varona C, Schwenck S, Sullivan MB. (2015). Variably lytic infection dynamics of large Bacteroidetes podovirus phi38:1 against two Cellulophaga baltica host strains. Environ Microbiol 17: 4659-4671.

Dang VT, Sullivan MB. (2014). Emerging methods to study bacteriophage infection at the single-cell level. Front Microbiol 5: 724.

Doron S, Fedida A, Hernandez-Prieto MA, Sabehi G, Karunker I, Stazic D et al. (2015). Transcriptome dynamics of a broad host-range cyanophage and its hosts. ISME J; e-pub ahead of print 1 December 2015; doi:10.1038/ismej.2015.210.

Duhaime MB, Sullivan MB. (2012). Ocean viruses: Rigorously evaluating the metagenomic sample-to-sequence pipeline. Virology 434: 181-186.

Falkowski PG, Fenchel T, Delong EF. (2008). The microbial engines that drive Earth's biogeochemical cycles. Science 320: 1034-1039.

Fallico V, Ross RP, Fitzgerald GF, Mcauliffe O. (2011). Genetic response to bacteriophage infection in $\mathrm{LaC}$ tococcus lactis reveals a four-strand approach involving induction of membrane stress proteins, D-alanylation of the cell wall, maintenance of proton motive force, and energy conservation. J Virol 85: 12032-12042.

Fuhrman JA. (1999). Marine viruses and their biogeochemical and ecological effects. Nature 399: 541-548.

Goldfarb T, Sberro H, Weinstock E, Cohen O, Doron S, Charpak-Amikam Y et al. (2015). BREX is a novel phage resistance system widespread in microbial genomes. EMBO J 34: 169-183.

Gomez-Pereira PR, Fuchs BM, Alonso C, Oliver MJ, Van Beusekom JE, Amann R. (2010). Distinct flavobacterial communities in contrasting water masses of the north Atlantic Ocean. ISME J 4: 472-487.

Heintz C, Mair W. (2014). You are what you host: microbiome modulation of the aging process. Cell 156: 408-411.

Holmfeldt K, Howard-Varona C, Solonenko N, Sullivan MB. (2014). Contrasting genomic patterns and infection strategies of two co-existing Bacteroidetes podovirus genera. Environ Microbiol 16: 2501-2513.
Holmfeldt K, Middelboe M, Nybroe O, Riemann L. (2007). Large variabilities in host strain susceptibility and phage host range govern interactions between lytic marine phages and their Flavobacterium hosts. Appl Environ Microbiol 73: 6730-6739.

Holmfeldt K, Solonenko N, Shah M, Corrier K, Riemann L, Verberkmoes NC et al. (2013). Twelve previously unknown phage genera are ubiquitous in global oceans. Proc Natl Acad Sci USA 110: 12798-12803.

Hurwitz BL, Hallam SJ, Sullivan MB. (2013). Metabolic reprogramming by viruses in the sunlit and dark ocean. Genome Biol 14: R123.

Hyman P, Abedon ST. (2012). Smaller fleas: viruses of microorganisms. Scientifica (Cairo) 2012: 734023.

Karlsson F, Malmborg-Hager AC, Albrekt AS, Borrebaeck CA. (2005). Genome-wide comparison of phage M13infected vs. uninfected Escherichia coli. Can J Microbiol 51: 29-35.

Kirchman DL. (2002). The ecology of Cytophaga-Flavobacteria in aquatic environments. FEMS Microbiol Ecol 39: 91-100.

Koerner JF, Snustad DP. (1979). Shutoff of host macromolecular synthesis after T-even bacteriophage infection. Microbiol Rev 43: 199-223.

Lavigne R, Lecoutere E, Wagemans J, Cenens W, Aertsen A, Schoofs L et al. (2013). A multifaceted study of Pseudomonas aeruginosa shutdown by virulent podovirus LUZ19. MBio 4: e00061-13.

Legendre M, Audic S, Poirot O, Hingamp P, Seltzer V, Byrne D et al. (2010). mRNA deep sequencing reveals 75 new genes and a complex transcriptional landscape in Mimivirus. Genome Res 20: 664-674.

Lin X, Ding H, Zeng Q. (2015). Transcriptomic response during phage infection of a marine cyanobacterium under phosphorus-limited conditions. Environ Microbiol.

Lindell D, Jaffe JD, Coleman ML, Futschik ME, Axmann IM, Rector $\mathrm{T}$ et al. (2007). Genome-wide expression dynamics of a marine virus and host reveal features of co-evolution. Nature 449: 83-86.

Lobocka MB, Rose DJ, Plunkett G III, Rusin M, Samojedny A, Lehnherr $\mathrm{H}$ et al. (2004). Genome of bacteriophage P1. J Bacteriol 186: 7032-7068.

Loskutoff DJ, Pene JJ, Andrews DP. (1973). Gene expression during the development of Bacillus subtilis bacteriophage phi 29. I. Analysis of viral-specific transcription by deoxyribonucleic acid-ribonucleic acid competition hybridization. J Virol 11: 78-86.

Luo Y, Li B-Z, Liu D, Zhang L, Chen Y, Jia B et al. (2015). Engineered biosynthesis of natural products in heterologous hosts. Chem Soc Rev 44: 5265-5290.

Mann NH, Clokie MR, Millard A, Cook A, Wilson WH, Wheatley PJ et al. (2005). The genome of S-PM2, a 'photosynthetic' T4-type bacteriophage that infects marine Synechococcus strains. J Bacteriol 187: 3188-3200.

Meyer F, Paarmann D, D'souza M, Olson R, Glass EM, Kubal M et al. (2008). The metagenomics RAST server - a public resource for the automatic phylogenetic and functional analysis of metagenomes. BMC Bioinform 9: 386.

Miller ES, Kutter E, Mosig G, Arisaka F, Kunisawa T, Ruger W. (2003). Bacteriophage T4 genome. Microbiol Mol Biol Rev 67: 86-156; table of contents.

Mojica KD, Brussaard CP. (2014). Factors affecting virus dynamics and microbial host-virus interactions in marine environments. FEMS Microbiol Ecol 89: 495-515. 
Mortazavi A, Williams BA, Mccue K, Schaeffer L, Wold B. (2008). Mapping and quantifying mammalian transcriptomes by RNA-Seq. Nat Methods 5: 621-628.

Omcallister WT, Wu HL. (1978). Regulation of transcription of the late genes of bacteriophage T7. Proc Natl Acad Sci USA 75: 804-808.

Pavlova O, Lavysh D, Klimuk E, Djordjevic M, Ravcheev DA, Gelfand MS et al. (2012). Temporal regulation of gene expression of the Escherichia coli bacteriophage phiEco32. J Mol Biol 416: 389-399.

Pene JJ, Murr PC, Barrow-Carraway J. (1973). Synthesis of bacteriophage phi 29 proteins in Bacillus subtilis. J Virol 12: 61-67.

Poranen MM, Ravantti JJ, Grahn AM, Gupta R, Auvinen P, Bamford DH. (2006). Global changes in cellular gene expression during bacteriophage PRD1 infection. J Virol 80: 8081-8088.

Prehm P, Jann K. (1976). Enzymatic action of coliphage omega8 and its possible role in infection. J Virol 19: 940-949.

Ravantti JJ, Ruokoranta TM, Alapuranen AM, Bamford DH. (2008). Global transcriptional responses of Pseudomonas aeruginosa to phage PRR1 infection. J Virol 82: 2324-2329.

Rice P, Longden I, Bleasby A. (2000). EMBOSS: the European Molecular Biology Open Software Suite. Trends Genet 16: 276-277.

Rinke C, Schwientek P, Sczyrba A, Ivanova NN, Anderson IJ, Cheng JF et al. (2013). Insights into the phylogeny and coding potential of microbial dark matter. Nature 499: 431-437.

Robinson MD, Mccarthy DJ, Smyth GK. (2010). edgeR: a Bioconductor package for differential expression analysis of digital gene expression data. Bioinformatics 26: 139-140.

Rohwer F, Segall AM. (2015). In retrospect: a century of phage lessons. Nature 528: 46-48.

Rossmann MG, Mesyanzhinov VV, Arisaka F, Leiman PG. (2004). The bacteriophage T4 DNA injection machine. Curr Opin Struct Biol 14: 171-180.

Roux S, Brum J, Dutilh BE, Sunagawa S, Duhaime MB, Loy A et al. (2015a). Ecogenomics of uncultivated globally abundant ocean viruses. Nature (in review). doi:10.1101/053090.

Roux S, Hallam SJ, Woyke T, Sullivan MB. (2015b). Viral dark matter and virus-host interactions resolved from publicly available microbial genomes. Elife 4: e08490.

Salmond GP, Fineran PC. (2015). A century of the phage: past, present and future. Nat Rev Microbiol 13: 777-786.

Samson JE, Magadan AH, Sabri M, Moineau S. (2013). Revenge of the phages: defeating bacterial defences. Nat Rev Microbiol 10: 675-687.

Shao Y, Wang IN. (2008). Bacteriophage adsorption rate and optimal lysis time. Genetics 180: 471-482.
Sharp PM, Li W-H. (1987). The codon adaptation index-a measure of directional synonymous codon usage bias, and its potential applications. Nucleic Acids Res 15: 1281-1295.

Smolkin M, Ghosh D. (2003). Cluster stability scores for microarray data in cancer studies. BMC Bioinform 4: 36 .

Solonenko SA, Ignacio-Espinoza JC, Alberti A, Cruaud C, Hallam S, Konstantinidis K et al. (2013). Sequencing platform and library preparation choices impact viral metagenomes. BMC Genom 14: 320.

Stocker R. (2012). Marine microbes see a sea of gradients. Science 338: 628-633.

Storms ZJ, Brown T, Cooper DG, Sauvageau D, Leask RL. (2014). Impact of the cell life-cycle on bacteriophage T4 infection. FEMS Microbiol Lett 353: 63-68.

Suzuki R, Shimodaira H. (2006). Pvclust: an R package for assessing the uncertainty in hierarchical clustering. Bioinformatics 22: 1540-1542.

Walsh D, Mohr I. (2011). Viral subversion of the host protein synthesis machinery. Nat Rev Microbiol 9: 860-875.

Wang IN. (2006). Lysis timing and bacteriophage fitness. Genetics 172: 17-26.

Weinbauer MG. (2004). Ecology of prokaryotic viruses. FEMS Microbiol Rev 28: 127-181.

Weitz JS, Stock CA, Wilhelm SW, Bourouiba L, Coleman ML, Buchan A et al. (2015). A multitrophic model to quantify the effects of marine viruses on microbial food webs and ecosystem processes. ISME $J$ 9: 1352-1364.

Wommack KE, Colwell RR. (2000). Virioplankton: Viruses in aquatic ecosystems. Microbiol Mol Biol Rev 64: 69-114.

You L, Suthers PF, Yin J. (2002). Effects of Escherichia coli physiology on growth of phage $\mathrm{T} 7$ in vivo and in silico. J Bacteriol 184: 1888-1894.

Zeglin LH. (2015). Stream microbial diversity in response to environmental changes: review and synthesis of existing research. Front Microbiol 6: 454.

(1) () This work is licensed under a Creative ar no Commons Attribution-NonCommercialNoDerivs 4.0 International License. The images or other third party material in this article are included in the article's Creative Commons license, unless indicated otherwise in the credit line; if the material is not included under the Creative Commons license, users will need to obtain permission from the license holder to reproduce the material. To view a copy of this license, visit http://creativecommons.org/ licenses/by-nc-nd/4.0/

Supplementary Information accompanies this paper on The ISME Journal website (http://www.nature.com/ismej) 